\title{
Dies und das über die Sprachen der Welt
}

Ernst Kausen: Die Sprachfamilien der Welt. Teil I: Europa und Asien. Hamburg: Buske 2013. XXXV + $1052 \mathrm{~S}$.

In diesem massiven, schon wegen seiner physischen Größe kaum handhabbaren Werk versucht Ernst Kausen, Professor für Mathematik und Theoretische Informatik an der Technischen Hochschule Mittelhessen, aber nebenbei auch Altorientalist und Ägyptologe, das (fast) Unmögliche. Er will sowohl die große, gefährdete und dem breiten Publikum leider sehr wenig bekannte Sprachendiversität der Welt vorstellen als auch die Strukturen und Grammatiken aller Sprachfamilien zumindest ansatzweise beschreiben. Das Buch richtet sich an das große Publikum, vor allem an sogenannte „sprachinteressierte Laien“. Vom Zielpublikum wird offensichtlich ein geradezu leidenschaftliches Interesse verlangt, denn die Leserschaft wird nicht nur vom gewaltigen Umfang des Buches herausgefordert, auch der Stil ist etwas schulmeisterhaft, sehr sachlich und trocken.

Nach der kurzen Einführung in das Phänomen Sprachverwandtschaft (Stammbäume, Rekonstruktion usw.), Sprachtypologie und Sprachareale folgt einer der besten Abschnitte des Buches: eine globale Sprachenstatistik mit einer detaillierten Einführung in die Problematik von Statistiken dieser Art, v.a. die Frage der Identifizierung von Sprachen. Es gibt ja bekanntlich Sprachvarietäten, deren Status (Sprache oder Dialekt?) sogar unter den Sprechern selbst umstritten ist. In diesem Abschnitt werden auch die soziolinguistischen $\mathrm{Me}$ chanismen der Sprachgefährdung und des Sprachtodes behandelt, wieder sachlich und überzeugend. Leider hat der Verfasser eine typische Gefahr nicht ganz vermeiden können: Wie so oft, kann auch hier der Eindruck entstehen (auch wenn 
der Verfasser nichts Solches explizit behauptet), der Sprachtod sei keine Folge von komplexen politischen und individuellen Entscheidungen, sondern quasi ein natürlicher, unvermeidlicher Prozess.

Nach einem kurzen Überblick über die Sprachfamilien Eurasiens folgen dann die Kapitel zu den einzelnen Sprachfamilien oder Sprachgruppen von Indogermanisch bis zu den Tai-Kadai-Sprachen, auch inklusive Isolatsprachen (Baskisch, Buruschaski) sowie Zusammenbündelungen von (oft nur schwach dokumentierten) Sprachen wie z. B. die altmediterranen, paläosibirischen und altorientalischen Sprachen. In ein einziges Kapitel sind auch „Altaische Sprachen, Koreanisch und Japanisch" zusammengebündelt; der Autor hegt große Sympathien für die makrophyletischen Ideen von Joseph Greenberg und seinen Schülern, auch wenn er korrekterweise zugibt, dass die genetische Verwandtschaft dieser Sprachen nicht endgültig bewiesen oder von der wissenschaftlichen Community einstimmig angenommen worden ist.

Die Kapitel zu den einzelnen Sprachfamilien sind nach dem üblichen Handbuchformat aufgebaut (vielleicht mit Ausnahme des Indogermanischen, denn zu dieser Familie gibt es schon ein ähnliches massives Handbuch vom gleichen
Autor, so dass hier auf eine ausführliche grammatische Darstellung verzichtet worden ist). Das heißt: Nach einer Einführung in die Geschichte, geographische Verbreitung und dialektale Einteilung der betreffenden Sprache(n) folgt eine grammatische Skizze: Phonologie, Morphologie und Syntax, mit besonderer Berücksichtigung der charakteristischen Merkmale (so wie beim Baskischen die Ergativität).

Im Folgenden werde ich mich auf die Kritik des uralischen Teiles beschränken, wobei sich natürlich die Frage ergibt, ob die Darstellung aller anderen Sprachfamilien und Gruppen auch unter ähnlichen Problemen leidet. Der Verfasser hat einen ehrlichen Versuch gemacht, sich in die Literatur einzuarbeiten. Falls er auch FinnougristInnen konsultiert hat, werden ihre Namen nicht erwähnt; auf die „zahlreichen Wissenschaftler", die den Verfasser in Einzelfragen beraten haben, wird in der Einführung nur kurz hingewiesen. Trotzdem hat er sich zumindest in der Uralistik nicht ganz auf den neuesten Wissensstand bringen können.

Schon bei der Einführung muss ein/e finnougristisch ausgebildete/r LeserIn ein paar Mal die Stirn runzeln: Saamisch wird zu den ostseefinnischen Sprachen eingeordnet (S. 6), und Mansisch soll (Harald Haarmann zufolge) den Wortstel- 
lungstyp OSV vertreten (S. 21). Die grundlegende Zweiteilung der uralischen Sprachfamilie in Samojedisch und Finnougrisch wird nicht bezweifelt, dagegen aber soll die "genetische Einheit" des finnougrischen Zweiges „in jüngster Zeit bestritten werden" (S. 49). Der Inhalt dieser kryptischen Aussage wird dann im uralischen Kapitel (S. 341370) erläutert. Teilweise handelt es sich um das „hyperkritische“ Werk von Angela Marcantonio (2002), wobei der Verfasser gleich anmerkt, dass ihre Ergebnisse wegen massiver methodischer Fehler zurückgewiesen werden, teilweise darum, dass der Verfasser dem Diskurs innerhalb der Uralistik nicht ganz folgen kann.

In seinem Vergleich verschiedener Stammbaummodelle wirft Kausen mehr und weniger seriöse Taxonomien, völlig veraltete (Austerlitz und Sauvageot in den 196oer7oer Jahren postulierten noch einen mordwinisch-marischen Zweig) und etwas jüngere (das hyperkritische Kamm- oder Buschmodell von Salminen 2002) in einen Topf. Dabei will er vermutlich seine Lieblingsthese von eurasischen makro- oder interphyletischen Anknüpfungen (die ja auch von Marcantonio hervorgehoben werden) dadurch untermauern, dass er die "genetische Einheit" des Finnougrischen bezweifelt. Ob unter „ge- netischer Einheit" die Verwandtschaft, die Abstammung aus einer gemeinsamen Grundsprache oder das Vorhandensein einer allgemein angenommenen Taxonomie gemeint wird, bleibt (absichtlich?) unklar. Dass „ein präzises, konsensfähiges Bild der internen Gliederung des Uralischen ferner denn je“ sei, kann nur jemand behaupten, der die neuesten Publikationen zu diesem Thema (s. z. B. Häkkinen 2009, Parpola 2012) nicht kennt.

In seinem Überblick über die uralischen Sprachen gibt der Verfasser ein sehr pessimistisches Bild von der Lage der kleinsten Minderheiten: wieder wird als quasi wissenschaftliche Tatsache dargestellt, dass sich „die heute noch vorhandene Vielfalt des Uralischen in wenigen Jahrzehnten auf die Großsprachen Ungarisch, Finnisch, Estnisch, Mordwinisch, Mari, Udmurtisch und Komi reduzieren" wird. Auch Nordsaamisch und Tundranenzisch wären demgemäß zum Tode verurteilt, so wie Karelisch, von welchem ,etliche Dialekte [welche?] schon ausgestorben" sind?

Die Darstellung der Geschichte und Gegenwart der uralischen Sprachen ist leider nicht frei von ärgerlichen Sachfehlern. Z. B. behauptet der Verfasser, dass die estnische Sprache schon Ende des 19. Jahrhunderts als Amts- und Schulsprache mit Russisch und 
Deutsch gleichgestellt wurde - in Wirklichkeit war eben das Ende des 19. Jahrhunderts die Zeit einer gewaltsamen Russifizierung vieler Institutionen. Auch glaubt er, dass Wepsisch, Chantisch und Mansisch (!) sowie Karelisch - in kyrillischer (!) Schrift - heute in Grundschulen als Unterrichtssprachen verwendet werden. Dies ist leider nicht einmal bei größeren fiu. Sprachen Russlands der Fall. Ebenso ist die Behauptung, dass die großen fiu. Minderheitssprachen im europäischen Russland „als Amtssprache" verwendet werden, leider eher Wunschdenken als Wirklichkeit, auch wenn Sprachgesetze in den fiu. Republiken die offizielle Verwendung der Nationalsprachen im Prinzip ermöglichen. Den Unterschied zwischen „Karelisch“ als Sprache/Ethnizität und „karelisch“ als regionale Identität innerhalb des finnischen Linguo-Ethnikums hat der Verfasser nicht ganz verstanden: von den 300.000 „Kareliern“, die „nach dem Krieg nach Finnland emigrierten" (d.h.: vor der sowjetischen Invasion systematisch evakuiert wurden), sprach in Wirklichkeit nur ein kleiner Bruchteil die karelische Sprache.

Unter den sprachlichen Charakteristika werden zuerst lexikalische Gemeinsamkeiten dargestellt, veranschaulicht mit einigen etymologischen Entsprechungen im
Grundwortschatz - mit Hinweis auf das Uralische Etymologische Wörterbuch, obwohl die Rekonstruktion der uralischen Protoformen (z. B. mit „Laryngal“ ${ }^{\star} x$ ) eher der von Janhunen und Sammallahti vertretenen Tradition entspricht. Von den kurz erwähnten lexikalen Kontakten zwischen Uralisch und Indogermanisch hat der Verfasser eine seltsame Auffassung: laut ihm stellen die uralischen ${ }^{\star} k a l a$,Fisch ${ }^{\circ}$ und ${ }^{\star}$ sexni [sic], Ader, Sehne' Lehnwörter aus dem Germanischen (!) dar.

Nach den Wortbeispielen folgen kurze Reflexionen über das Ururalische. Hier nimmt der Verfasser die Kritik von Marcantonio für bare Münze: für die uralische Grundsprache sei keine umfassende Morphologie rekonstruierbar, und deshalb stelle sich die Frage, ob die in der Indogermanistik entwickelte komparativ-historische Methode in der Uralistik überhaupt anwendbar sei. (Wenn nicht, wie kann man überhaupt die uralische Sprachgeschichte erforschen?) Dabei ist ja ein großer Teil der Suffixe uralt und wirklich für das Protouralische rekonstruierbar, obwohl die Rekonstruktion von ganzen Subsystemen der Nominal- und Verbalmorphologie immer noch viele offene Fragen enthält.

Im darauffolgenden Abschnitt werden phonologische Merkmale 
kurz dargestellt: Die Konsonantensysteme des Ururalischen, Finnischen (hier wird wieder der Mythos vom phonematischen Glottalklusil weitergegeben) und Ungarischen in Tabellen, sowie die Vokalinventare des Finnischen, Ungarischen, Nenzischen und "Chantischen“ (ohne Angabe des Dialekts) einfach aufgelistet. Die Vokalharmonie sei kein proto-uralisches Merkmal, "eher liegt ein altaischer Einfluss vor" (auch im Ostseefinnischen?!), und auch die „Konsonantenstufung" (Stufenwechsel) sei eher eine saamisch-finnische Innovation, weil es umstritten sei (!), ob in den samojedischen Sprachen „Spuren der Konsonantenstufung zu finden sind".

Von der Morphologie werden zuerst die Nominalflexion (Numerus, Kasus und Possessivsuffixe), dann die Deklination der Personalpronomina und zuletzt die Verbalmorphologie mit Tabellen von Paradigmen illustriert und kurz kommentiert. Die Beispiele stammen zumeist aus dem Finnischen und Ungarischen, für einige Kategorien auch aus dem Nenzischen. Der Versuch, auf 10 Seiten ein kohärentes Gesamtbild der uralischen Morphologie zu geben, ist selbstverständlich zum Scheitern verurteilt, zumal sehr viel Platz für Flexionstabellen und etwas auch für Erörterungen von makrophyleti- schen eurasischen Anknüpfungen (ein Steckenpferd des Verfassers) verwendet wird.

Die meisten Tabellen und Auskünfte werden direkt aus Handbüchern zitiert, mitunter begegnen aber etwas seltsame Kommentare und Interpretationen. Z. B. wird unter „sekundärer Kasusbildung“ auch die Grammatikalisation von Postpositionen im Ungarischen kurz erwähnt (was nur indirekt dazugehört und das nichteingeweihte Publikum wahrscheinlich nur verblüffen kann), und unter "komplexer Nominalphrase" wird die Reihenfolge von Suffixen behandelt. Von den Tempora (hier seltsamerweise „Tempus-Aspekt“ genannt) werden die Kategorien Präsens(-Futur), Imperfekt, Perfekt, Plusquamperfekt erwähnt; unwissende LeserInnen werden in dem Glauben bleiben, dass diese Kategorien überall im Uralischen vorhanden sind (und eventuell, dass „Imperfekt“ und „Perfekt“ auch aspektuale Kategorien darstellen). Für die Objektkonjugation werden nur ungarische Beispiele gegeben, mit den irreführenden Termini „definite“ und „indefinite Konjugation" und ohne Hinweis auf die komplexen Objektmarkierungssysteme in anderen uralischen Sprachen. Zuletzt werden von der Syntax des Uralischen nur „Negation und Besitz" in einem 
minimal kurzen Abschnitt geschildert. Der (prädikative?) Besitz soll „meist durch ,sein' mit dem Dativ und Adessiv" ausgedrückt werden; vom Gebrauch der Possessivsuffixe also kein Wort (geschweige denn von den habeo-Verben des Obugrischen), und auch das ungarische Beispiel ohne Possessivsuffix (Jánosnak van egy ház (sic) ,János hat ein Haus') wirkt sehr seltsam.

Fazit: Der Verfasser hat mit lobenswerter Mühe aus teilweise veraltetem, dubiösem oder missverstandenem Handbuchwissen eine Sammlung von Fakten und Beispielen zusammengebastelt, um die interne Diversität der Sprachfamilie, vor allem die großen Unterschiede zwischen Finnisch und Ungarisch, zu illustrieren. Als Nichtfachmann kann er aber die genetischen und arealen Beziehungen - mit Ausnahme von vagen Vermutungen über inter- oder makrophyletische eurasische Verbindungen - nicht ordentlich erläutern, es entsteht kein zusammenhängendes Bild. Die nichteingeweihten LeserInnen stehen hilflos vor diesem sehr lückenhaften Mosaik und müssen einfach alles für bare Münze nehmen - die Auskünfte überprüfen können sie nicht, denn obwohl ab und zu auf das Handbuch von Abondolo (Hg. 1998) sowie auf das schon ziemlich veraltete Hajdú \& Domokos (deutsche Auflage 1987) hingewiesen wird, bleiben eben die seltsamsten Behauptungen (so wie die über Karelisch mit kyrillischer Schrift als Schulsprache oder die germanischen Lehnwörter im Uralischen) ohne Quellenangabe. Aus den ambitiösen Zielen des Buches ergibt sich das vielleicht größte Problem: Der Verfasser kann sich keine unterhaltenden Ausschweifungen erlauben, alles muss extrem kurz, ex cathedra, festgestellt werden, und für das nichteingeweihte Publikum entsteht ein Trugbild von absoluter Expertise und wissenschaftlicher Zuverlässigkeit.

Zumindest das uralische Kapitel enthält so viel Missverstandenes, Verzerrtes oder Veraltetes, dass man es einem Laien als „Einführung in die uralischen Sprachen" nicht in die Hand geben kann. Falls sich, wie zu befürchten ist, die gleichen Probleme auch in den anderen Kapiteln wiederholen, muss man den Sinn dieses kühnen Publikationsvorhabens ernsthaft hinterfragen. Lohnt es sich wirklich, eine auch für Laien verständliche Darstellung der weltweiten Sprachendiversität mit einem „Handbuch aller Sprachen der Welt“ zu kombinieren - wäre es nicht besser gewesen, die extrem aktuellen und wichtigen Fragen der Sprachenvielfalt und Sprachgefährdung, und warum nicht auch der Sprachverwandtschaft und Sprachgeschichte, 
in einem kompakteren und handlicheren Werk zu behandeln, mit ausgewählten Beispielen und ohne den Anspruch, alle Sprachfamilien der Welt abzudecken? Und warum hat der Verlag, dem der Verfasser „für das große Vertrauen“ dankt und der sich schon stark in der Uralistik profiliert hat, die Auskünfte über die uralischen Sprachen nicht von ExpertInnen überprüfen lassen?

Johanna Laakso

\section{Literatur}

Abondolo, Daniel (Hg.) 1998: The Uralic languages. LondonNew York: Routledge.

Hajdú, Péter \& Péter Domokos 1987: Die uralischen Sprachen und Literaturen. Hamburg: Buske.
HäKкINEN, JAAKко 2009: Kantauralin ajoitus ja paikannus. Perustelut puntarissa. - JSFOu 92: 9-56. <http:// www.sgr.fi/susa/92/hakkinen.pdf $>$.

Marcantonio, Angela 2002: The Uralic language family: Facts, myths, and statistics. Oxford: Blackwell.

Parpola, Asko 2012: Formation of the Indo-European and Uralic (FinnoUgric) language families in the light of archaeology: Revised and integrated 'total' correlations. - Riho Grünthal \& Petri Kallio (Hgg.): A linguistic map of prehistoric Northern Europe. MSFOu 266. Helsinki: Suomalais-Ugrilainen Seura.

Salminen, Tapani 2002: Problems in the taxonomy of the Uralic languages in the light of modern comparative studies. - Лингвистический беспредел: сборник статей к то-летию А. И. Кузнецовой. Москва: Издательство Московского университета. 44-55. <http://www. helsinki.fi/ tasalmin/kuzn.html>. 